\title{
Acknowledgment to Reviewers of Fractal and Fractional in 2020
}

\section{Fractal and Fractional Editorial Office}

Citation: Fractal Fract Editorial

Office. Acknowledgment to

Reviewers of Fractal and Fractional in

2020. Fractal Fract. 2021, 5, 12.

https://doi.org/10.3390/

fractalfract5010012

Published: 28 January 2021

Publisher's Note: MDPI stays neutral with regard to jurisdictional claims in published maps and institutional affiliations.

Copyright: (c) 2021 by the author. Licensee MDPI, Basel, Switzerland. This article is an open access article distributed under the terms and conditions of the Creative Commons Attribution (CC BY) license (http://creativecommons.org/licenses /by/4.0/).
MDPI AG, St. Alban-Anlage 66, 4052 Basel, Switzerland

Peer review is the driving force of journal development, and reviewers are gatekeepers who ensure that Fractal and Fractional maintains its standards for the high quality of its published papers. Thanks to the cooperation of our reviewers, in 2020, the median time to first decision was 40 days and the median time to publication was 19 days. The editors would like to express their sincere gratitude to the following reviewers for their precious time and dedication, regardless of whether the papers were finally published:

\begin{tabular}{|c|c|}
\hline Abdel-Haleem, Abdel-Aty & Claire, David \\
\hline Ahmed S., Elwakil & Corina, Drapaca \\
\hline Ahmed, Madian & Costas, Psychalinos \\
\hline Ahmet, Akdemir & D. G, Prakasha \\
\hline Alejandro Pérez, Riascos & Dana, Copot \\
\hline Aleksei, Chechkin & Danko D., Georgiev \\
\hline Alexander J., Bies & Dario, Magliacano \\
\hline Ali, Ahmadian & David, Kubanek \\
\hline Ali, Kurt & Da-Yan, Liu \\
\hline Aly, Seadawy & Dimiter, Prodanov \\
\hline Amar, Debbouche & Diogo Francisco, Rossoni \\
\hline Amin, Jajarmi & Dmitrii, Tumakov \\
\hline Andrea, Cugno & Dusan, Zorica \\
\hline Andrey, Zahariev & Emile Franc, Doungmo Goufo \\
\hline Andrzej, Dzieliński & Enrica, Pirozzi \\
\hline Andrzej, Horzela & Erdal, Karapinar \\
\hline Angelica, Malaspina & Erhan, Set \\
\hline António M., Lopes & Ernesto, Zambrano-Serrano \\
\hline Antonio, Gonzalez & Ervin K., Lenzi \\
\hline Arran, Fernandez & Fan, Zhang \\
\hline Ashraf Mohamed, Tawfik & Federico, Cluni \\
\hline Ayed, Ale'damat & Gissell, Estrada-Rodriguez \\
\hline Babak, Shiri & Guido, Maione \\
\hline Bakhtiyor Jalilovich, Kadirkulov & H. T., Liu \\
\hline Bhuvaneswari, Sambandham & Haci Mehmet, Baskonus \\
\hline Bongsoo, Jang & Haruhiko, Ogasawara \\
\hline Boris, Baeumer & Hossein, Jafari \\
\hline Bruce, Henry & Igor, Podlubny \\
\hline Bruno, Lombard & Inés, Tejado \\
\hline C., Park & Irina, Petreska \\
\hline Camillo, Porcaro & Iulia Martina, Bulai \\
\hline Carla, Pinto & Ivailo, Pandiev \\
\hline Chun Kit, Lai & Jae-Hun, Jung \\
\hline
\end{tabular}


Jalil, Manafianheris

Jan, Glaubitz

Jaroslav, Koton

Javier, Gallegos

Jean-Claude, Trigeassou

Jean-Daniel, Djida

Jeevani, Jayasinghe

Ji-huan, $\mathrm{He}$

Jiqiang, Jiang

Jocelyn, Sabatier

Joel Esteban, Restrepo Tangarife

Jordan, Hristov

Jorge, Sanchez-Ortiz

José Francisco, Gómez Aguilar

José, Sánchez-Dehesa

Juan Gabriel, Avina-Cervantes

Juan, Nieto

Kai, Diethelm

Kai, Liu

Khaled, Mehrez

Kobra, Rabiei

Kolade M., Owolabi

Kottakkaran Sooppy, Nisar

Krystian, Czernek

Kyongok, Kang

Lester, Ingber

Luciano, Mescia

Luisa, Beghin

Mahmoud, Abdel-Aty

Manuel, Fernández-Martínez

Manuel, Ortigueira

Marcel, Bogdan

Marcelo, Lenzi

Marek, Rydel

Maria Alessandra, Ragusa

Marina, Yashina

Marko, Vrdoljak

Martin, Stynes

Mehmet, Yavuz

Mesut, ŞEKER

Michael, Chryssomallis

Michał, Awtoniuk

Michele, Sciacca

Mikhail, Kalmykov

Ming, Li

Mircea, Dulău

Mirko, D'Ovidio

Mokhtar, Kirane

Monika, Rybczak

Mostafa M. A., Khater

Muhammad, Farooq
Mustafa, Inc

Nazim I, Mahmudov

Ndolane, Sene

Necati, Ozdemir

Nguyen, Xuan-Mung

Nickolay, Korabel

Nikolay K, Vitanov

Nimisha, Pathak

Otman, Mrabet

Patrick D., Shipman

Péter, Ván

Rabha W., Ibrahim

Raegan, Higgins

Ravi, Ravichandran

Renat T., Sibatov

Ricardo, Almeida

Richard, Herrmann

Roberto, Garrappa

Roman, Kubacki

Roman, Sotner

Ryszard, Sikora

Salvatore, Graziani

Sameer Chakravarthy, Vedula

Samira, Hamani

Sansit, Patnaik

Santos, Bravo-Yuste

Sergiy, Koshkin

Shy-Der, Lin

Snezhana, Hristova

Soheil, Salahshour

Stanislav, Lukashchuk

Stefano, Serra Capizzano

Sverre, Holm

Tamara, Radivilova

Teodor, Atanackovic

Themistokles, Rassias

Thomas, Takacs

Tomasz P., Stefański

Tommaso, Alberti

Trevor, Langlands

Trifce, Sandev

Tuomas, Yrttimaa

Valeriy, Martynyuk

Vasiliki, Vita

Vasily E., Tarasov

Vassili, Kolokoltsov

Vaughn, Voller

Vicenç, Méndez

Victor, Bakhtin

Vsevolod, Bohaienko

Waleed, Abdelhamed 
William S., Oates

Wojciech J., Krzysztofik

Wojciech, Sumelka

Wookyong, Kwon

Xuefeng , Zhang

YangQuan, Chen

Yanping, Chen

Yiheng, Wei

Yiming, Chen
Yong, Wang

Youssri H., Youssri

Yulong, Li

Yung, Ting

Yuriy, Povstenko

Zakia, Hammouch

Zhiping, Mao

Zhiyuan, Li 